Pacific Journal of Mathematics

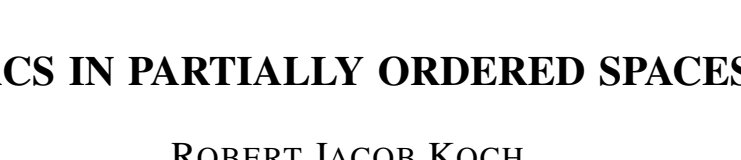




\section{ARCS IN PARTIALLY ORDERED SPACES}

\section{R. J. $\mathrm{KoCH}$}

We present here a theorem on the existence of arcs in partially ordered spaces, and several applications to topological semigroups. The hypotheses are motivated by the structure of the partially ordered set of principal ideals of a compact connected topological semigroup with unit. Noteworthy among the applications are $(1)^{1}$ a compact, connected topological semigroup with unit contains an arc. (2) A compact, connected topological semigroup with zero, each of whose elements is idempotent, is arc-wise connected. Throughout the paper, arc is used in the sense of "continuum irreducibly connected between two points". We do not assume metricity of the spaces, but all spaces are assumed to be Hausdorff. Simple non-metric examples of the theorems are furnished by the "long line", i.e. the ordinals up to and including $\Omega$, filled in with intervals, the operation being $a \cdot b=\min (a, b)$. The author is indebted to R. D. Anderson, R. P. Hunter, and W. Strother for useful suggestions.

We recall the following definitions: $[10](X, \leq)$ is a partially ordered space if $X$ is a space, and $\leq$ is a reflexive, antisymmetric, transitive binary relation on $X$. A chain in $X$ is an ordered subset of $(X, \leq)$. We denote by Graph $(\leq)$ the set of pairs $(x, y)$ with $x \leq y$. We denote by $A \backslash B$ the complement of $B$ in $A$; closure is denoted by*, $F(A)$ denote the boundary of $A$, and $\square$ denotes the empty set.

The following result of the author [3] is presented here in detail because of its relation with Theorem 2 .

THEOREM 1. Let $(X, \leq)$ be a compact partially ordered space and let $W$ be an open set in $X$. If

(1) For each $x \in X,\{y \mid y \leq x\}$ is closed, and

(2) For any $x \in W$, each open set about $x$ contains an element $y$ with $y<x$,

then if $C$ is any component of $W, C^{*} \cap F(W) \neq \square$.

Proof. We show first that if $V$ is open and $V \subset W$, then $F(V) \neq$ $\square$. Let $M$ be a maximal chain in $V^{*}$. Then $M$ is compact [10], hence $M$ has a minimal element $m \in V^{*}$. If $m \in V$, then by hypothesis (2) above, the chain $M$ can be extended, contrary to the maximality. Now

Received September 4, 1958. This research was supported by the United States Air Force through the Air Force Office of Scientific Research, Air Research and Development Command, under Contract AF 18 (603)-89.

* 1This settles a question raised by D. Montogomery. The author learned of the question through A. D. Wallace. 
let $C$ be a component of $W$ and suppose $C^{*} \cap F^{\prime}(W)=1$. Then by standard arguments [5; p. 110] there is an open and closed set $N$ with $C \subset N \subset W$. Hence $N$ is an open subset of $W$ with $F(N)=\square$, a contradiction.

In the next theorem we use the following topology for the space $S(X)$ of non empty closed subsets of a compact space $X$, which is an extension of the Hausdorff metric topology [1]. For open sets $U$ and $V$ of $X$, let $N(U, V)=\{A \mid A \in S(X), A \subset U, A \cap V \neq L]\}$. Take $\{N(U, V) \mid U, V$ open\} for a subbasis for the open sets of $S(X)$. It is known that if $X$ is compact Hausdorff, so is $S(X)$.

Theorem 2. Let $(X, \leq)$ be a compact partially ordered space, and let $W$ be an open set in $X$. If

(1) Graph $(\leq)$ is closed and

(2) For any $x \in W$, each open set about $x$ contains an element $y$ with $y<x$,

then any element $x$ of $W$ belongs to a (compact) connected chain $C$ with $C \cap F(W) \neq \square$ and $x=\sup C$.

Proof. Let $W$ be as above, and fix $a \in W$. Since $W^{*} \cap\{y \mid y \leq a\}$ is a compact partially ordered space and contains the relatively open set $W \cap\{y \mid y \leq a\}$ satisfying the above hypotheses, we may assume that $X=\{y \mid y \leq a\}$ and that $W$ is an open set in $X$ with $a \in W \subset W^{*}=X$. Let $\mathscr{C}$ denote the collection of all closed chains in $X$ with $a \in C$ and $C \cap F(W) \neq \square$. By Theorem 1, $F(W) \neq \square$; hence if $z \in F(W)$, the elements $\alpha$ and $z$ constitute an element of $\mathscr{C}$, so that $\mathscr{C} \neq \square$.

(i) $\mathscr{C}$ is closed in $S(X)$. We show that $S(X) \backslash \mathscr{C}$ is open. Let $A$ be closed in $X$, with $A \notin \mathscr{C}$. If $A$ is not a chain, then there are elements $x$ and $y$ of $A$ with $x \not \leq y$ and $y \not \leq x$. By hypothesis (1) there exist open sets $U$ and $V$ about $x$ and $y$ with the property that $x^{\prime} \in U$, $y^{\prime} \in V$ imply $x^{\prime} \not \leq y^{\prime}$ and $y^{\prime} \not \leq x^{\prime}$. Then $N(X, U) \cap N(X, V)$ is an open set about $A$, and misses $\mathscr{C}$. If $A$ is a chain but $a \notin A$, then $(X \backslash a, X \backslash a)$ is an open set about $A$ which misses ' $\mathcal{C}$, and the case $F(W) \cap A=\square$ goes in a similar way.

Define $L(x)=\{y \mid y \leq x\}$ and $M(x)=\{y \mid x \leq y\}$. Also define $(x, y)=$ $\{z \mid x<z<y\}$. Let $\delta$ be an open cover of $X$, and define a subset $M_{\delta}$ of $S(X)$ by: $C \in M_{\delta}$ iff $C$ is a closed chain in $X$, and for any $x$ and $y$ in $C^{\prime}$ with $x<y$ and $(x, y) \cap C=\square$, there exists $V \in \delta$ such that $V^{*}$ meets both $L(x) \cap C$ and $M(y) \cap C$.

(ii) $M_{\delta} \cap \epsilon^{\prime} \neq$, for any open cover $\delta$. Let $\delta$ be an open cover of $X$, let $\mathscr{L}$ be the collection of all closed chains $C$ with $C \subset W, C \in M_{\delta}$, 
and $a \in C$. Let $\mathscr{f}$ be a maximal tower in $\mathscr{D}$, and let $T=\bigcup \mathscr{f}$. Note that $T$ is a chain containing $a$, hence by hypothesis (1), $T^{*}$ is again a chain. Also it is easily seen that $\inf T=\inf T^{*}$. We will show that $T^{*} \in M_{\delta}$. Let $x, y \in T^{*}$ with $x<y$ and $(x, y) \cap T^{*}=\square$. Suppose $x=\inf T$; then if $\inf T \notin T$ it follows that $T \subset M(y)$, hence $x \in T^{*} \subset M(y)$, a contradiction. Hence $x=\inf T \in T$, so there exists $T_{1} \in \mathscr{F}$ such that $x \in T_{1}$. Since $T_{1} \in M_{\delta}$, there is $V \in \delta$ with $V^{*}$ meeting both $L(x) \cap T_{1}$ and $M(y) \cap T_{1}$. Hence $V^{*}$ meets both $L(x) \cap T^{*}$ and $M(y) \cap T^{*}$, and it follows that $T^{*} \in M_{\delta}$. Therefore we may suppose $\inf T<x<y$, so there exists $t_{0}$ with $\inf T<t_{0} \leq x<y, t_{0} \in T_{0} \in \mathcal{F}$. Since $T_{0} \in M_{\delta}$, it again follows that $T^{*} \in M_{\delta}$. This establishes that $T^{*} \in M_{\dot{\delta}}$ and it remains to show $T^{*} \in \mathscr{C}$. If $T^{*} \subset W$, then $T^{*} \in \mathscr{D}$, so by the maximality of $T, T^{*}=T$. Hence $\inf T \in T \subset W$, so there exists $V \in \delta$ with $\inf T \in V$. There is an element $y \in V \cap W$ with $y<x$. By an easy argument, $T \cup y \in M_{\delta}$, so $T \cup y \in \mathbb{X}, T \cup y=T$, and $y \in T$, a contradiction. We conclude that $T^{*} \cap F(W) \neq \square$, and $T^{*} \in M_{\delta} \cap \mathcal{C}^{\text {. }}$

(iii) $M_{\delta} \cap \mathscr{C}$ is closed for each finite open cover $\delta$ of $X$. Let $\delta$ be a finite open cover of $X$. We show that $S(X) \backslash M_{i} \cap \mathscr{C}$ is open. Let $A$ be a closed set, $A \notin M_{\delta} \cap \mathscr{C}$. Then either $A \notin \mathscr{C}$, or $A \in \mathscr{C}$ but $A \notin M_{\delta}$. If $A \notin \mathscr{C}$, then by part (i) there is an open set about $A$ which misses $\mathscr{C}$. Suppose $A \in \mathscr{C}$ and $A \notin M_{\delta}$. Then for some $x, y \in A$ we have $(x, y) \cap A=\square$, and for each $V \in \delta, V^{*}$ misses either $L(x) \cap A$ or $M(y) \cap A$. By an easy argument which makes use of hypothesis (1) and compactness, there are open sets $U_{1}$ and $W_{1}$ about $L(x) \cap A$ and $M(y) \cap A$ resp. with the property that $x^{\prime} \in U_{1}$ and $y^{\prime} \in W_{1}$ imply $y^{\prime} \not \leq x^{\prime}$. Let $U_{2}=\bigcap\left\{X \backslash V^{*} \mid V \in \delta, V^{*} \cap L(x) \cap A=\square\right\}, W_{2}=\bigcap\left\{X \backslash V^{*} \mid V \in \delta\right.$, $\left.V^{*} \cap M(y) \cap A=\square\right\}$. Then, since $\delta$ is finite, $U_{2}$ is open and contains $L(x) \cap A, W_{2}$ is open and contains $M(y) \cap A$, and for each $V \in \delta, V^{*}$ misses either $U_{2}$ or $V_{2}$. Let $U^{\prime}=U_{1} \cap U_{2}, W^{\prime}=W_{1} \cap W_{2}$. Now $N\left(U^{\prime} \cup W^{\prime}, U^{\prime} \cup W^{\prime}\right)$ is open, contains $A$, and as we next show, misses $M_{\delta} \cap \mathscr{C}$. Suppose $C \in \mathscr{C} \cap N\left(U^{\prime} \cup W^{\prime}, U^{\prime} \cup W^{\prime}\right)$. Let $x^{\prime}=\sup \left(C \cap U^{\prime}\right), y^{\prime}=$ $\inf \left(C \cap W^{\prime}\right)$; then $\left(x^{\prime}, y^{\prime}\right) \cap C=\square$. Also $L\left(x^{\prime}\right) \cap C \subset U^{\prime \prime}$ and $M\left(y^{\prime}\right) \cap C \subset W^{\prime}$, and since for each $V \in \delta, V^{*}$ misses either $U^{\prime}$ or $W^{\prime}$, we conclude that $C \notin M_{\delta}$. This completes (iii).

For any finite open cover $\delta$, put $P_{\delta}=M_{\delta} \cap \mathscr{C}$, and let $\mathscr{P}=\left\{P_{\delta}\right\}$. Note that if $P_{\alpha}, P_{\beta} \in \mathscr{P}$, then there is a finite open cover $\gamma$ which refines both $\alpha$ and $\beta$, and hence $P_{\gamma} \subset P_{\alpha} \cap P_{\beta}$. Therefore $\bigcap \mathscr{P} \neq \square$. Let $C \in \cap \mathscr{P}$, and we show next that $C$ is order dense. Let $x, y \in C$ with $x<y$. Then $L(x) \cap M(y) \cap C=\square$, so by normality there are open sets $U$ and $V$ about $L(x) \cap C$ and $M(y) \cap C$ resp., with $U^{*} \cap V^{*}=\square$.

Let $\alpha$ be the finite open cover consisting of $\left\{X \backslash U^{*}, X \backslash V^{*}\right\}$. Since $C \in P_{\alpha}$ and it is false that the closure of each member of $\alpha$ meets both 
$L(x)$ and $M(y)$, we conclude that $(x, y) \cap C \neq \square$, and $C$ is order-dense. Hence $C$ is a compact order dense chain from $a$ to $F(W)$, and is therefore an arc. The proof is complete.

CoRollary 1. Let $(X, \leq)$ be a compact partially ordered space with unique minimal element 0 . If

(1) Graph ( $\leq$ ) is closed in $X \times X$ and

(2) $L(x)$ is connected for each $x \in X$, then $X$ is arcwise connected.

Proof. Let $W=X \backslash \mid 0$; then from the connectedness of each $L(x)$, we see that $W$ satisfies the hypotheses of Theorem 2. Hence each element of $X$ can be joined to 0 by a compact connected chain.

We note that Corollary 1 contains a result of Wallace [8].

Let $S$ be a compact topological semigroup, and for $a \in S$, let $J(a)=$ $a \cup S a \cup a S \cup S a S$, and let $J=\{(x, y) \mid J(x)=J(y)\}$. Endow $\frac{S}{J}$ with the quotient topology, and let $\rho: S \rightarrow \frac{S}{J}$ be the natural mapping. From the compactness of $S$ it follows that $\frac{S}{J}$ is compact Hausdorff.

We denote by $E$ the set of idempotents of $S$.

COROLlaRY 2. Let $S$ be a compact connected topological semigroup, with $S=E S \cup S E$ : then $\frac{S}{J}$ is arcwise connected.

Proof. We define a partial order in $\frac{S}{J}$ by : $\varphi(a) \leq \varphi(b)$ iff $J(a) \subset J(b)$. From the compactness of $S$ it follows that Graph $(\leq)$ is closed in $S \times S$. Further, if $K$ denotes the minimal ideal of $S$, then $\varphi(K)$ is the unique minimal element of $\frac{S}{J}$. Note that $L(\varphi(x))=J(x)$; since $S=E S \cup S E$, it follows that $J(x)$ is connected. Hence Corollary 1 applies, and the proof is complete.

REMARK. By a similar argument it can be seen that if $L$ is a continuous monotone reflexive struct [4.7] on a continuum $X$, all of whose minimal elements are related, then $\frac{X}{L}$ is arcwise connected.

Corollary. 3. Let $S$ be a compact connected topological semigroup with unit $u$, and let $V$ be an open set about $u$; then $V$ contains an arc.

Proof. If $S$ is a group, the result is known [2], hence we may suppose that $u \notin K$, where $K$ is the minimal ideal of $S$ [9]. Let $E$ denote 
the set of idempotents of $S$, and partially order $E$ by: $e \leq f$ iff $e \in f S f$. It follows from the compactness of $S$ that Graph $(\leq)$ is closed in $E \times E$. If there exists an open set $W$ about $u$ such that $W \cap E$ satisfies (2) of Theorem 2 (taking $X=E$ ), then $W \cap V \cap E$ also satisfies (2), so there is an arc in $V$. If for each open set $W$ about $u, W \cap E$ fails to satisfy (2), then there exists $e \in E \cap V \cap(S \backslash K)$ such that $e S e \cap E \cap V=\{e\}$. Since $e \notin K, e S e$ is non-degenerate. Hence $e S e$ is a compact connected semigroup with unit $e$ in which there is an open set containing $e$ but no other idempotent. By a theorem of Mostert and Shields [6] there is a local one-parameter semigroup in $e S e \cap V$ and the proof is complete.

A compact connected semigroup with unit may fail to contain an arc which contains the unit. This is illustrated in Example 2 below, and is due to R. P. Hunter (unpublished).

EXAMPLE 1 . Let $R_{+}$denote the non-negative reals under addition, and let $C$ by the unit disc in the complex plane: $C=\{z:|z| \leq 1\}$ Let $W=\left\{(z, t) ; z=\exp (2 \pi i s), t=e^{-s}, s \in R_{+}\right\}$, and set $S=(C \times\{0\}) \cup W$. Then $S$ is a compact connected semigroup with zero and unit, but does not contain a standard thread joining the two. We may describe $S$ by saying it is a two-cell with an arc winding on its boundary.

ExAMPLE 2. Let $D_{i}$ be the graph of $x^{2}+y^{2} \leq \frac{1}{i} ; z=1-\frac{1}{i} ; i=$ $1,2,3, \cdots$. The $D_{i}$ then converge to a point $u$. From the center of $D_{i+1}$ we start an arc $A_{i}$ which winds upon the boundary of $D_{i}$ as in Example 1. Let $S_{i}=A_{i} \cup D_{i} ; S_{i}$ is then a compact connected semigroup with zero $0_{i}$ and unit $u_{i}$. Repeat this construction for each positive integer $i$, and let $S=\mathrm{U}_{i} S_{i}$. We define multiplication in $S$ as follows: if $x, y \in S$ and both $x$ and $y$ belong to the same $S_{i}$, let $x y$ be the product

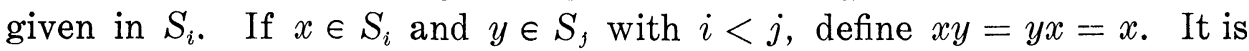
easy to see that $S^{*}$ becomes a compact connected semigroup with zero $0_{1}$ and unit $u$. Moreover, no arc in $S^{*}$ contains $u$.

COROLlary 4. Let $S$ be a locally compact connected topological semigroup with zero (0), each of whose elements is idempotent; then $S$ is arcwise connected.

Proof. Note that $e \leq f$ iff $e \in f S f$ defines a partial order on $S$, and that Graph $(\leq)$ is closed in $S \times S$. The conclusion is now immediate from Corollary 1.

We conjecture that compactness can be replaced by local compactness in Corollary 4, and further, that a locally compact connected semilattice is arcwise connected. 


\section{REFERENCES}

1. O. Frink, Topology in lattices, Trans. Amer. Math. Soc., 51 (1942), 569-582.

2. A. M. Gleason, Arcs in locally compact groups, Proc. Nat. Acad. Sci., 36 (1950), 663-667.

3. R. J. Koch, Note on weak cutpoints in clans, Duke Math. J., 24 (1957), 611-616.

4. I. S. Krule, Structs on the 1-sphere, Duke Math. J., 24 (1957), 405-414.

5. C. Kuratowski, Topologie, II, Warsaw, 1950.

6. P. S. Mostert and A. L. Shields, One parmeter semigroups in a semigroup, (to appear).

7. A. D. Wallace, Struct ideals, Proc. Amer. Math. Soc., 6 (1955), 634-638.

8. - Partial order and indecomposability, Proc. Amer. Math. Soc., 5 (1954), $780-781$.

9. … The structure of topological semigroups, Bull. Amer. Math. Soc., 61 (1955), 95-112.

10. L. E. Ward, Jr. Partially ordered topological spaces, Proc. Amer. Math. Soc., 5 (1954), 144-161.

LOUISIANA STATE UNIVERSITY 


\section{PACIFIC JOURNAL OF MATHEMATICS}

\section{EDITORS}

\section{David Gilbarg}

Stanford University Stanford, California

\section{R. A. Beaumont}

University of Washington

Seattle 5, Washington

\section{A. L. Whiteman}

University of Southern California Los Angeles 7, California

L. J. Paige

University of California

Los Angeles 24, California

\section{ASSOCIATE EDITORS}

E. F. BECKENBACH
C. E. BURGESS
E. HEWITT
A. HORN

A. HORN

\author{
V. GANAPATHY IYER \\ R. D. JAMES \\ M. S. KNEBELMAN \\ L. NACHBIN
}

I. NIVEN

T. G. OSTROM

H. L. ROYDEN

M. M. SCHIFFER
E. G. STRAUS

G. SZEKERES

F. WOLF

K. YOSIDA

\section{SUPPORTING INSTITUTIONS}

\author{
UNIVERSITY OF BRITISH COLUMBIA \\ CALIFORNIA INSTITUTE OF TECHNOLOGY \\ UNIVERSITY OF CALIFORNIA \\ MONTANA STATE UNIVERSITY \\ UNIVERSITY OF NEVADA \\ OREGON STATE COLLEGE \\ UNIVERSITY OF OREGON \\ OSAKA UNIVERSITY \\ UNIVERSITY OF SOUTHERN CALIFORNIA
}

\author{
STANFORD UNIVERSITY \\ UNIVERSITY OF TOKYO \\ UNIVERSITY OF UTAH \\ WASHINGTON STATE COLLEGE \\ UNIVERSITY OF WASHINGTON \\ * * * * \\ AMERICAN MATHEMATICAL SOCIETY \\ CALIFORNIA RESEARCH CORPORATION \\ HUGHES AIRCRAFT COMPANY \\ SPACE TECHNOLOGY LABORATORIES
}

Mathematical papers intended for publication in the Pacific Journal of Mathematics should be typewritten (double spaced), and the author should keep a complete copy. Manuscripts may be sent to any one of the four editors. All other communications to the editors should be addressed to the managing editor, L. J. Paige at the University of California, Los Angeles 24, California.

50 reprints per author of each article are furnished free of charge; additional copies may be obtained at cost in multiples of 50 .

The Pacific Journal of Mathematics is published quarterly, in March, June, September, and December. The price per volume (4 numbers) is $\$ 12.00$; single issues, $\$ 3.50$. Back numbers are available. Special price to individual faculty members of supporting institutions and to individual members of the American Mathematical Society: $\$ 4.00$ per volume; single issues, $\$ 1.25$.

Subscriptions, orders for back numbers, and changes of address should be sent to Pacific Journal of Mathematics, 2120 Oxford Street, Berkeley 4, California.

Printed at Kokusai Bunken Insatsusha (International Academic Printing Co., Ltd.), No. 6, 2-chome, Fujimi-cho, Chiyoda-ku, Tokyo, Japan.

PUBLISHED BY PACIFIC IOURNAL OF MATHEMATICS, A NON-PROFIT CORPORATION

The Supporting Institutions listed above contribute to the cost of publication of this Journal, but they are not owners or publishers and have no responsibility for its content or policies. 


\section{Pacific Journal of Mathematics}

\section{Vol. 9, No. $3 \quad$ July, 1959}

Errett Albert Bishop, A minimal boundary for function algebras . . . . . . . . . . . . 629

John W. Brace, The topology of almost uniform convergence . . . . . . . . . . . . 643

Cecil Edmund Burgess, Chainable continua and indecomposability .......... 653

L. Carlitz, Multiplication formulas for products of Bernoulli and Euler

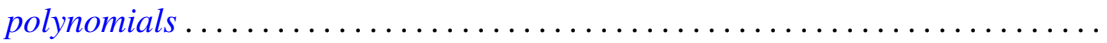

Eckford Cohen, A class of residue systems (mod $r$ ) and related arithmetical

functions. II. Higher dimensional analogues ....................

Shaul Foguel, Boolean algebras of projections of finite multiplicity . . . . . . . . . .

Richard Robinson Goldberg, Averages of Fourier coefficients .................

Seymour Goldberg, Ranges and inverses of perturbed linear operators .

Philip Hartman, On functions representable as a difference of convex functions ....

Milton Vernon Johns, Jr. and Ronald Pyke, On conditional expectation and

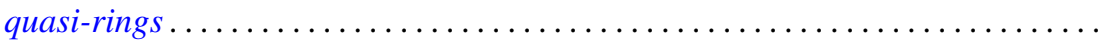

Robert Jacob Koch, Arcs in partially ordered spaces ....................

Gregers Louis Krabbe, A space of multipliers of type $L^{p}(-\infty, \infty) \ldots \ldots \ldots \ldots$

John W. Lamperti and Patrick Colonel Suppes, Chains of infinite order and their

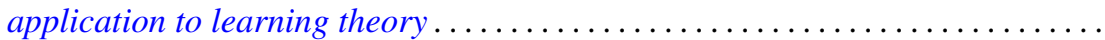

Edith Hirsch Luchins, On radicals and continuity of homomorphisms into Banach

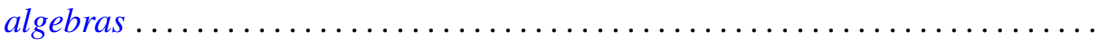

T. M. MacRobert, Multiplication formulae for the E-functions functions of their parameters.

Michael Bahir Maschler, Classes of minimal and representative domains and their kernel functions.

William Schumacher Massey, On the imbeddability of the real projective spaces in Euclidean space.

Thomas Wilson Mullikin, Semi-groups of class $\left(C_{0}\right)$ in $L_{p}$ determined by parabolic differential equations

Steven Orey, Recurrent Markov chains

Ernest Tilden Parker, On quadruply transitive groups ........ . .

Calvin R. Putnam, On Toeplitz matrices, absolute continuity, and unitary

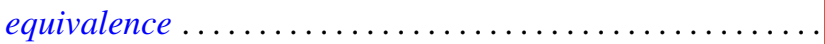

Helmut Heinrich Schaefer, On nonlinear positive operators.

Robert Seall and Marion Wetzel, Some connections between continued fractions and convex sets

Robert Steinberg, Variations on a theme of Chevalley

Olga Taussky and Hans Zassenhaus, On the similarity transformation between a

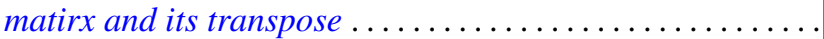

Emery Thomas, The suspension of the generalized Pontrjagin cohomology

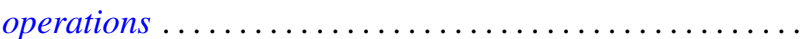

Joseph L. Ullman, On Tchebycheff polynomials ..................... 913

Richard Steven Varga, Orderings of the successive overrelaxation scheme ........ 925

Orlando Eugenio Villamayor, Sr., On weak dimension of algebras . 